\title{
Human Papillomavirus-Related Multiphenotypic Sinonasal Carcinoma with Late Recurrence
}

\author{
Bokyung Ahn, Eojin Kim, Harim Oh, Yang-Seok Chae, Chul Hwan Kim, Youngseok Lee, Jeong Hyeon Lee, Yoo Jin Lee \\ Department of Pathology, Korea University Anam Hospital, Korea University College of Medicine, Seoul, Korea
}

In 2013, Bishop et al. ${ }^{1}$ introduced a newly-described human papillomavirus (HPV)-related sinonasal carcinoma, referred to as "HPV-related carcinoma with adenoid cystic carcinoma-like features," and it is provisionally included in the 2017 World Health Organization (WHO) classification of head and neck tumors. ${ }^{2}$ In 2017, Bishop et al. ${ }^{3}$ suggested a change in the name to 'HPV-related multiphenotypic sinonasal carcinoma' to encompass its broader histologic features. ${ }^{1}$ We report a case of recurrent 'HPV-related multiphenotypic sinonasal carcinoma' with the second-longest follow-up time and an unusual HPV genotype with abrupt keratinization.

\section{CASE REPORT}

A 66-year-old man underwent surgical resection for a polypoid lesion in his right nasal cavity, and he was diagnosed with adenoid cystic carcinoma, solid variant. He received no additional postoperative treatment, and he did not have any routine follow-up visits for 16 years until he presented to the Otorhinolaryngology Department of our institution due to 12 months of right nasal obstruction. A new fungating mass in the right nasal cavity was found, and a piecemeal removal was performed.

Microscopically, the tumor showed highly cellular proliferation of basaloid cells with a predominantly lobular growth pattern and comedonecrosis (Fig. 1A). There were several foci of cribriform growth pattern aligned around cylindromatous microcystic spaces filled with mucopolysaccharide-like basophilic

Received: January 29, 2019 Revised: March 27, 2019

Accepted: April 2, 2019

Corresponding Author: Yoo Jin Lee, MD

Department of Pathology, Korea University Anam Hospital, Korea University

College of Medicine, 73 Inchon-ro, Seongbuk-gu, Seoul 02841, Korea

Tel: +82-2-920-5590, Fax: +82-2-920-6576, E-mail: yujinn87@korea.ac.kr material, resembling adenoid cystic carcinoma (Fig. 1B, C). The tumor cells showed relatively large, uniform, round nuclei with prominent nucleoli and scant granular cytoplasm. Frequent squamous differentiation with abrupt keratinization (Fig. 1D) and varying degrees of dysplasia of the overlying surface squamous epithelium were noted (Fig. 1E). Neither lymphovascular nor perineural invasion was present. On immunohistochemical staining, the tumor cells were diffusely positive for cytokeratin and p63, but they were negative for CD117 immunostain in both the solid and cribriform areas. As a surrogate marker for the presence of HPV, p16 immunostain showed strong and diffuse reactivity in nearly the entire tumor, except the dysplastic surface squamous epithelium (Fig. 1F). We further confirmed the presence of HPV genotype 33 and 51 by quantitative HPVspecific polymerase chain reaction (Anyplex II HPV28 Detection, Seegene Inc., Seoul, Korea).

The combination of the microscopic findings, the immunoprofile, and the HPV testing results allowed us to reclassify the formerly diagnosed adenoid cystic carcinoma to the newly-emerging entity, HPV-related multiphenotypic sinonasal carcinoma.

\section{Ethics statement}

Study approval was obtained from the Institutional Review Board of Korea University Anam Hospital (IRB No. 2019AN 0012), and informed consent was waived.

\section{DISCUSSION}

Until now, 56 cases of HPV-related carcinoma with adenoid cystic carcinoma-like features have been reported. ${ }^{1,3-6}$ The tumor is defined as a sinonasal carcinoma with the morphologic features of a salivary gland tumor including admixed ductal and myoepithelial elements, surface squamous epithelial dysplasia, 

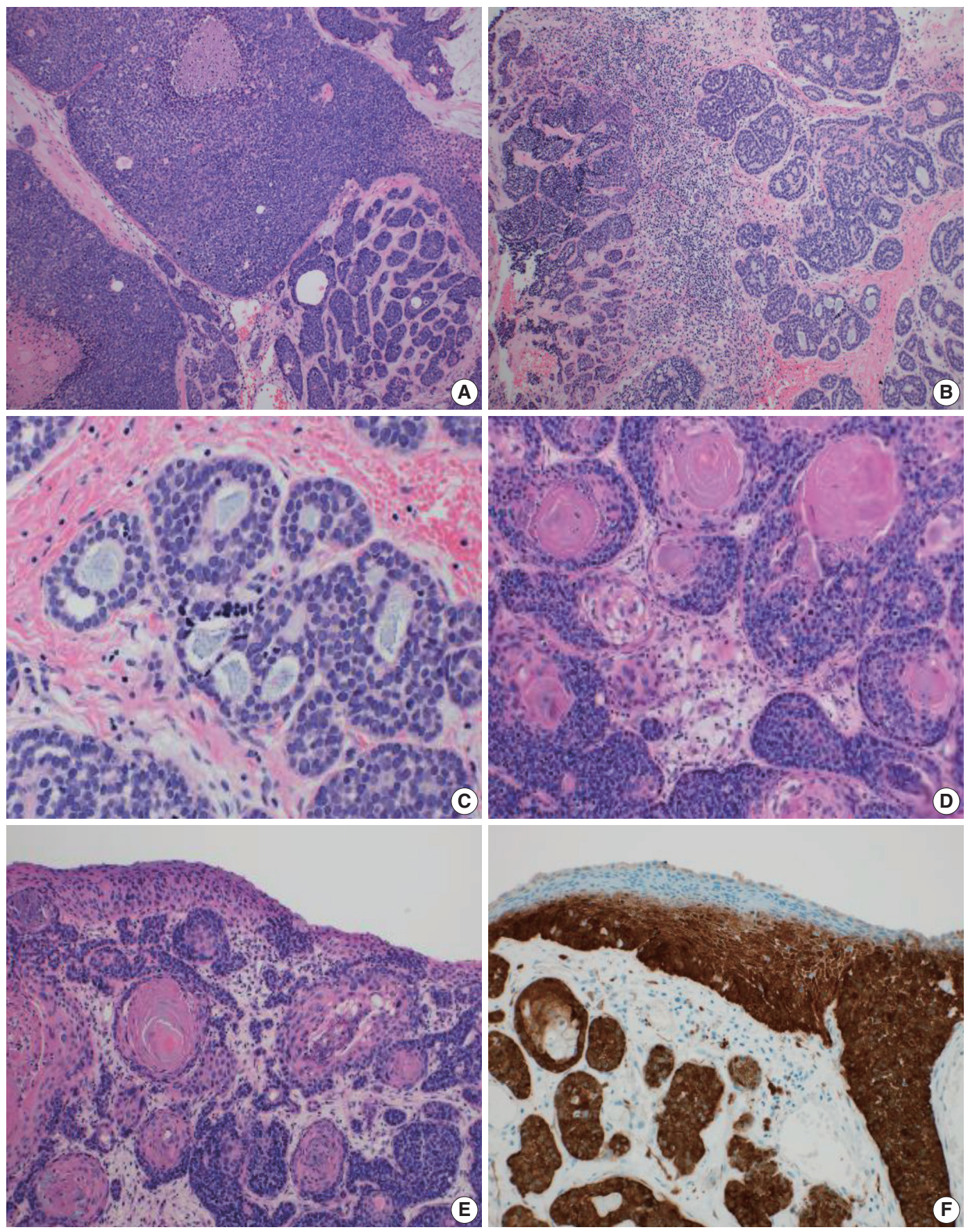

Fig. 1. Microscopic findings of an human papillomavirus-related multiphenotypic sinonasal carcinoma. (A) Basaloid cells with predominant solid, lobular pattern and comedonecrosis. (B, C) A few foci of cribriform architecture resembling adenoid cystic carcinoma. (D) Frequent squamous differentiation with abrupt keratinization. (E) Varying degree of surface epithelial dysplasia. (F) Diffuse, strong positive p16 immunostain sparing the dysplastic surface epithelium.

association with high-risk HPV (particularly type 33), and no MYB gene fusion. A new terminology, HPV-related multiphenotypic sinonasal carcinoma, has been recently suggested due to the tumor's diverse morphology, including anaplastic giant cells, clear cells, sarcomatoid cells, chondroid structure, osseous differ- entiation, and hemangiopericytoma-like blood vessels.

Although this tumor was classified under the non-keratinizing squamous cell carcinoma category in the 2017 WHO classification of head and neck tumors, only a few cases showed squamous differentiation and abrupt keratinization. ${ }^{2,3,7}$ A total 
of nine cases demonstrated scattered foci of squamous differentiation, ${ }^{3}$ with two cases showing abrupt keratinization, ${ }^{8}$ and our case is another with abrupt keratinization. Given these various morphological features, keratinization alone cannot be a differential point from conventional squamous cell carcinoma.

This tumor was suggested to have an indolent behavior with a better prognosis than other carcinomas. ${ }^{3,6}$ While the reported mean follow-up time is 42 months, frequent local recurrence (13 patients), rare distant metastasis (2 patients), and no tumor-related death were noted. ${ }^{1,3-6}$ One former report introduced a recurrent case after a 30 -year disease-free interval, ${ }^{6}$ and our case has the second-longest follow-up of any published reports, supporting the tumor's characterization of being indolent yet having potential for late recurrence. Due to the small number of reported cases and limited clinical follow-up, more clinical data are needed to better understand the tumor prognosis and treatment options.

Another interesting point of our case is that, to our knowledge, this is the first case of HPV-related multiphenotypic sinonasal carcinoma that showed the high-risk HPV genotype 51. HPV 51 is included in the WHO-categorized cancer-causing HPV types and is a high-risk carcinogen in head and neck cancer. ${ }^{9}$ However, its oncogenic implication or the tumor characteristics associated with HPV 51 infection have not been determined. One study reported HPV 51 to be one of the predominant genotypes in squamous cell carcinoma of the oral cavity and larynx, ${ }^{10}$ although none of the HPV-related multiphenotypic sinonasal carcinomas have shown HPV 51 thus far.

To better understand the clinicopathologic features of this rare disease, more data on such rare cases need to be collected. We report a case of HPV-related multiphenotypic sinonasal carcinoma with abrupt keratinization and a rare HPV type that recurred after a long indolent period of 16 years.

\section{ORCID}

Bokyung Ahn: https://orcid.org/0000-0002-0229-2276

Eojin Kim: https://orcid.org/0000-0003-3111-6754

Harim Oh: https://orcid.org/0000-0003-4904-4015

Yang-Seok Chae: https://orcid.org/0000-0002-8801-0910

Chul Hwan Kim: https://orcid.org/0000-0003-2026-8824

Youngseok Lee: https://orcid.org/0000-0002-9762-4957

Jeong Hyeon Lee: https://orcid.org/0000-0003-2041-4617

Yoo Jin Lee: https://orcid.org/0000-0003-3830-7051

\section{Author Contributions}

Conceptualization: BA.
Data curation: YJL.

Formal analysis: BA, YJL.

Investigation: $\mathrm{BA}, \mathrm{HO}, \mathrm{EK}$.

Methodology: JHL, YL, CHK, YSC.

Project administration: YJL, CHK.

Resources: YJL.

Supervision: YJL.

Validation: YJL, CHK.

Visualization: BA, YJL.

Writing—original draft: BA.

Writing—review \& editing: YJL.

\section{Conflicts of Interest}

The authors declare that they have no potential conflicts of interest.

\section{Funding}

No funding to declare.

\section{REFERENCES}

1. Bishop JA, Ogawa T, Stelow EB, et al. Human papillomavirus-related carcinoma with adenoid cystic-like features: a peculiar variant of head and neck cancer restricted to the sinonasal tract. Am J Surg Pathol 2013; 37: 836-44.

2. El-Naggar AK, Chan JK, Grandis JR, Takata T, Slootweg PJ. WHO classification of head and neck tumours. 4th ed. Lyon: IARC Press, 2017.

3. Bishop JA, Andreasen S, Hang JF, et al. HPV-related multiphenotypic sinonasal carcinoma: an expanded series of 49 cases of the tumor formerly known as HPV-related carcinoma with adenoid cystic carcinoma-like features. Am J Surg Pathol 2017; 41: 1690-701.

4. Chouake RJ, Cohen M, Iloreta AM. Case report: HPV-related carcinoma with adenoid cystic-like features of the sinonasal tract. Laryngoscope 2018; 128: 1515-7.

5. Ruangritchankul K, Jitpasutham T, Kitkumthorn N, Thorner PS, Keelawat S. Human papillomavirus-related multiphenotypic sinonasal carcinoma: first case report associated with an intermediaterisk HPV type and literatures review. Hum Pathol Case Rep 2018; 14: $20-4$.

6. Shah AA, Lamarre ED, Bishop JA. Human papillomavirus-related multiphenotypic sinonasal carcinoma: a case report documenting the potential for very late tumor recurrence. Head Neck Pathol 2018; 12: 623-8.

7. Bishop JA, Westra WH. Human papillomavirus-related multiphenotypic sinonasal carcinoma: An emerging tumor type with a unique 
microscopic appearance and a paradoxical clinical behaviour. Oral Oncol 2018; 87: 17-20.

8. Hang JF, Hsieh MS, Li WY, et al. Human papillomavirus-related carcinoma with adenoid cystic-like features: a series of five cases expanding the pathological spectrum. Histopathology 2017; 71: 887-96.
9. Kashif M, Afzal N, Tayyab MA, Shahzad F, Jahan S. Human papilloma virus: important etiological factor in head and neck squamous cell carcinogenesis. J Cancer Biol Res 2016; 4: 1081.

10. Snow AN, Laudadio J. Human papillomavirus detection in head and neck squamous cell carcinomas. Adv Anat Pathol 2010; 17: 394-403. 